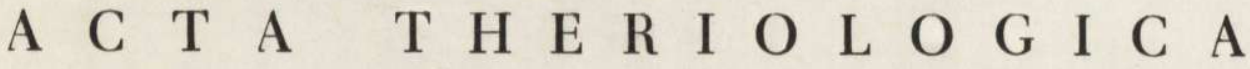 \\ VOL. XI, 9: 289-296. \\ BIAEOWIEŻA \\ 30.X.1966
}

Tadeusz ROSKOSZ \& Stanisław PYTEL

\section{Wirbel- und Rippenvariationen beim Rothirsch}

\author{
[Mit 1 Tabelle \& 2 Tafeln]
}

Gestützt auf 42 Hirschskelette (29 Hirschböcke u. 13 Hirschkühe) führten die Autoren zahlenmässige und morphologische Wirbelbeobachtungen der einzelnen Abschnitte der Wirbelsäule durch. Wirbelverschiebungen, die mit dem Angleichen an die Wirbel der benachbarten Abschnitte verbunden waren, wurden wie folgt bestimmt: Costalisatio des ersten Lendenwirbels (10 Individuen); Lumbalisatio des ersten Kreuzwirbels (1 Individuum); Sacralisatio des ersten Schwanzwirbels (11 Individuen). In diesen Abwandlungen, die bei Hirschen sehr oft vorkommen und unabhängig von Alter und Geschlecht auftreten, notierten wir eine grössere Anteilnahme der Hirschböcke.

\section{EINFÜHRUNG}

Die Bestimmung der Wirbelanzahl in den einzelnen Abschnitten der Wirbelsäule beim Säugetier kann auf Irrwege führen, wenn man über ein spärliches Untersuchungsmaterial verfügt. Auf eben diese Weise bestimmte C uvier (1825) die Wirbelanzahl beim Bison bison ( $\mathrm{L}$ in n a e u s, 1758), obwohl er nur eine Bisonkuh dieser Art zur Verfügung hatte. Wie es sich später erwies, waren die Angaben irrtümlich, aber sie überdauerten einige Jahrzehnte lang in dem anatomischen Schrifttum.

Lehrbücher entscheiden dieses Problem ebenfalls ziemlich oberflächlich. Gewöhnlich werden in ihnen Angaben über die Zahlenmässigkeit der Wirbel in den entsprechenden Abschnitten der Wirbelsäule oder auch die Amplitude der Anzahlschwankungen angeführt, wobei diese Angaben als physiologisch angenommen werden (K o ld a, 1936; P o plewski, 1948; Wokk en et al., 1961): Um Irrtümern vorzubeugen, wird die Wirbelanzahl auch für die beiden miteinander benachbarten Abschnitte bestimmt (A kajevski, 1962).

Es ist jedoch der Erwähnung wert, dass eine Reihe von Autoren eine gewisse Verschiebung in der Wirbelanzahl in Betracht zieht, nämlich eines Wirbels aus einem Abschnitt zugunsten des anderen, was natürlich Veränderungen in den morphologischen Merkmalen der verschobenen Wirbel nach sich zieht. Eine Úbersicht des Schrifttums aus diesem Gebiet enthält die Arbeit von Roskos z (1962). 
Man kann auch eine Erläuterungsprobe dieser Kontroverse beim Jagdwild zur Kenntnis nehmen. Interessante Positionen darüber sind: Die Arbeit von Bronn (1874), in der der Autor unter anderen die Wirbelanzahl in der Familie Cervidae auf eine ziemlich originelle Weise darstellt und die Mittelung von Pilarski \& Roskosz (1959) über das Thema des Rumpfabschnittes der Wirbelsäule beim Elch.

Ein zahlreiches Untersuchungsmaterial, wenn es sich um derartige Untersuchungen handelt, und das Fehlen von originellen Positionen in dieser Hinsicht, veranlassten uns dazu, sich der Reihe nach mit dem Problem der Wirbelanzahl bei den Vertreter der Familie der Cervidae zu befassen und zwar bei dem Rothirsch, Cervus elophus Linnaeus, 1758.

\section{MATERIAL UND METHODE}

Die Untersuchungen wurden auf Wirbelsäulen von 42 Hirschen verschiedenen Aiters und Geschlechts (29 Hirschböcke und 13 Hirschkühe) durchgeführt. 36 Skelette sind im Besitz des Institutes für Anatomie der Tiere der Landwirtschaftlichen Hochschule in Warszawa. Sie gehören zu Individuen, die aus dem Gebiet von Polen stammen. Nähere Angaben darüber enthält die Arbeit von Roskos z \& Pyte 1 (1966). Die übrigen 6 Skelette dagegen wurden in den wissenschaftlichen Instituten der UdSSR (Moskau, Charkow, Kiew) beobachtet.

Nach durchgeführter, genauer, vergleichender Durchsicht der Wirbelsäulen aller Exemplare, wurden zu weiteren Untersuchungen diejenigen Individuen abgesondert, bei denen zahlenmässig morphologische Veränderungen der Wirbel festgestellt worden waren. Ausser der Beschreibung dieser Fälle wurden ihre photographische Dokumentation und die Tabelle hergestellt, die ảie besprochenen Wirbelverschiebungen vortrefflich illustrieren. Der Halsabschnitt, Pars cervicalis, in dem keine Veränderungen festgestellt worden waren, wurde in den weiteren Erwägungen ausser Acht gelassen.

\section{EIGENE BEOBACHTUNGEN}

Im Brustabschnitt, Pars thoracalis, der Wirbelsäule des Hirsches notierten wir bei der Mehrzahl der Individuen beiderlei Geschlechts die Anwesenheit von 13 Wirbeln. In 8 Fällen jedoch vergrösserte sich diese Anzahl auf 14. Als Grundlage für diese Feststellung diente das Vorhandensein des vierzehnten Rippenpaares. Der genaue Befund führte zur Folgerung, dass hier eine Verschiebung des ersten Lendenwirbels zum Brustabschnitt eingetreten war, die eine weit gehende Angleichung dieses Wirbels mit den Brustwirbeln miteinbezogen hatte und zwar schon in Hinsicht auf die Vereinigung vermittels der Rippen. Es ist klar, dass sich in diesen Fällen die Wirbelanzahl des Lendenabschnittes um den verschobenen Wirbel vermindert. Die Adaptation des ersten Lendenwirbels war nicht in allen Fällen gleich.

Das zusätzliche vierzehnte Rippenpaar vereinigte sich folglich auf eine für die Brustwirbel typische Weise mit den Foveae costales caudales des dreizehnten Brustwirbels und den Foveae costales craniales des ersten 
Lendenwirbels. Diese Abwandlung stellten wir bei einem 10-jährigen Hirschbock (Kat. Nr. 2697), einer über 12 Jahre alten Hirschkuh (Kat. Nr. 383), einem erwachsenen Hirschbock (Kat. Nr 2 K) und einem 2,5jährigen Hirschbock (Kat. Nr. 480) fest. Bei den beiden letzten Individuen war neben der beschriebenen beiderseitigen Vereinigung vermittels des Rippenköpfchens, die Rippe noch ebenfalls beiderseits mit dem Rippenhöckerchen mit entsprechenden Flächen auf den Querfortsätzen des ersten Lendenwirbels vereinigt (Phot. 1, 2). Das vierzehnte Rippenpaar der besprochenen Individuen hatte ein für Costae arcuariae typisches Aussehen. Dafür zeugt - ihre Länge (zirka $25 \mathrm{~cm}$ ) und die rauhen Flächen auf den ventralen Endstücken, die zweifelsohne zur Vereinigung mit den Rippenknorpeln dienen; im Fall mit der Hirschkuh (Kat. Nr. 383) bewahrte sich sogar ein Fragment des Rippenknorpels der vierzehnten Rippe. Die Querfortsätze waren in den besprochenen Fällen um so viel verkürzt, dass man sie für Querfortsätze eines normalen Brustwirbels halten kann.

In den zwei anderen Fällen vereinigten sich die Rippenköpfchen des vierzehnten Paares sogar nur mit den Gelenkflächen, die auf den Querfortsätzen des ersten Lendenwirbels situiert waren. Diese Fortsätze unterlagen keiner wesentlicheren Verkürzung und die mit ihnen verbundenen Rippen schienen ebenfalls Costae arcuariae zu sein (Phot. 3). Derartige Verhältnisse traten bei zwei Hirschböcken auf: dem 5-jährigen (Kat. Nr. 463) und dem über 12-jährigen (Kat. Nr. 362).

Eine Zwischenstellung nimmt der 10-jährige Hirschbock (Kat. Nr. 381) ein. Bei ihm lässt sich nämlich die linke Rippe mit ihrem wirbelseitigen Endstück so zusammenstellen, wie dies bei den Hirschen (Nr. Nr. $2697 \mathrm{u}$. 383) beschrieben war, folglich mit dem letzten Brustwirbel und dem ersten Lendenwirbel, aber die rechte Rippe vereinigt sich nur mit dem Querfortsatz des ersten Lendenwirbels. Eine derartige Vereinigung verursachte eine deutliche Asymmetrie des ersten Lendenwirbels (Phot. 4).

Eine aparte Besprechung erfordern die Verhältnisse, die bei dem 10-jährigen Hirschbock (Kat. Nr. 375) festgestellt worden sind. Das vierzehnte Rippenpaar vereinigt sich zwar nur mit den Querfortsätzen des ersten Lendenwirbels, was auch bei den schon beschriebenen Hirschen stattfand, aber die Art und Weise der Vereinigung ist verschieden. Die rechte Rippe ist vermittels der Synostosis vereinigt und ist gewissermassen eine Verlängerung des rechten Querfortsatzes. Sie sieht prächtig aus und misst $22 \mathrm{~cm}$. An Statt des früheren Rippenhöckerchengelenkes tritt eine sichtbare Verdickung auf. Die linke Rippe ist mit dem ersten Lendenwirbel vermöge der rauhen Flächen vereinigt, die auf dem Querfortsatz dieses Wirbels und auf dem entsprechend erweiterten wirbelseitigen Endstück dieser Rippe situiert sind (Phot. 5). Diese Rippe ist von der 
anderseitigen schwächer, trotz ihrer grösseren Länge $(24 \mathrm{~cm})$. Der Charakter dieser Vereinigung scheint von seiner geringen Beweglichkeit zu zeugen.

Die oben beschriebenen 8 Adaptionsfälle des ersten Lendenwirbels an den Brustabschnitten kann man, obwohl die Art und Weise der Vereinigung der beiderseitigen Rippen mit diesen Wirbeln nicht bei allen Exemplaren gleich war, als Thoracalisatio des ersten Lendenwirbels bestimmen. Die adaptierten Wirbel muss man dagegen, aus Rücksicht auf das Beibehalten von gewissen Merkmalen der Lendenwirbel, als sogenannte Übergangswirbel betrachten.

Tabelle 1.

Zahlenmässige Wirbelvariationen in den Abschnitten der Wirbelsäule beim Hirsch

\begin{tabular}{|c|c|c|c|c|c|}
\hline $\begin{array}{l}\text { Kat. } \\
\text { Nr. }\end{array}$ & Geschlecht & $\begin{array}{c}\text { Alter } \\
\text { in Jahren }\end{array}$ & $\begin{array}{c}\text { Brust- } \\
\text { abschnitt }\end{array}$ & $\begin{array}{l}\text { Lenden- } \\
\text { abschnitt }\end{array}$ & $\begin{array}{l}\text { Kreuz- } \\
\text { abschnitt }\end{array}$ \\
\hline 480 & $0^{*}$ & 2,5 & $13+1$ & 5 & $4+1$ \\
\hline 463 & $0^{\circ}$ & 5 & $13+1$ & 5 & $4+1$ \\
\hline 381 & $c^{\circ}$ & 10 & $13+1$ & 5 & $4+1$ \\
\hline 375 & $c^{\circ}$ & 10 & $13+1$ & 5 & $4+1$ \\
\hline $2 \mathrm{~K}$ & $\sigma^{\prime \prime}$ & 10 & $13+1$ & 5 & 4 \\
\hline 2697 & $c^{\prime}$ & 10 & $13+1$ & $5+1$ & 4 \\
\hline 362 & $0^{\circ}$ & über 12 & $13+1$ & 5 & $4+1$ \\
\hline 383 & \& & über 12 & $13+1$ & 5 & 4 \\
\hline 361 & $c^{\prime}$ & 5 & $13+1^{*}$ & 5 & $4+1$ \\
\hline 364 & $0^{\prime}$ & über 12 & $13+1^{*}$ & 5 & $4+1$ \\
\hline 360 & $\sigma^{\prime}$ & 6 & 13 & 6 & $4+1$ \\
\hline 416 & q & 8 & 13 & 6 & $4+1$ \\
\hline 352 & $0^{\circ}$ & 11 & 13 & 6 & $4+1$ \\
\hline 351 & $0^{7}$ & über 12 & 13 & 6 & $4+1$ \\
\hline
\end{tabular}

*) einseitige Costalisatio

Zum Schluss unserer Erwägungen über den Brustabschnitt lohnt es sich noch von zwei nacheinander folgenden Individuen anzumerken, bei denen der erste Lendenwirbel sich an die Brustwirbel anähnelte, aber nur einseitig. Es handelt sich hier um folgende Hirschböcke: den 5-jährigen (Kat. Nr. 361) und den über 12-jährigen (Kat. Nr. 364). Im ersten Fall vereinigte sich dieser Wirbel durch eine weite Gelenkfläche auf dem verkürzten Querforsatz der rechten Seite mit der langen $(25 \mathrm{~cm})$ gut ausgebildeten Rippe; der linksseitige Querfortsatz behielt dagegen den typischen Bau für die Lendenwirbel (Phot. 6). Im zweiten Fall tritt ebenfalls eine Rippe auf, aber die linksseitige, wobei diese vermittels der Synostosis mit dem verkürzten und stark verdickten Querfortsatz des ersten Lendenwirbels verbunden ist, beim Beibehalten durch den Querfortsatz 
der gegenüberliegenden Seite einer für den Lendenwirbel rechtmässigen Gestalt (Phot. 7).

Die besprochenen Fälle, in denen die Adaptation des ersten Lendenwirbels an die Wirbel des Brustabschnittes auf das Auftreten nur einer Rippe, entweder der linken oder der rechten Seite reduziert werden kann, lassen sich als einseitige Thoracalisatio des ersten Lendenwirbels bestimmen.

$\mathrm{Da}$ alle bisher beschriebenen Fälle hauptsächlich auf dem Auftreten von zusätzlichen Rippen beruhen und die Vereinigung mit ihnen durch die Teilnahme des ersten Lendenwirbels bewirkt wird, möchten wir den bisher angewandten Termin „Thoracalisatio” durch den Begriff Costalisatio ersetzen. Dies erläutert auf eine offenbar ausdrückliche Weise die sich im Bereich des ersten Lendenwirbels vollziehenden Veränderungen und zeugt von der Variabilität des kaudalen Abschnittes des Brustkorbes.

Der Lendenabschnitt, Pars lumbalis, zählt beim Hirsch 6 Wirbel. Diese Anzahl unterliegt einer Verminderung bis fünf in denjenigen Fällen, von denen oben die Rede war, d.h. dann, wenn der erste Lendenwirbel die Gestalt des Brustwirbels annimmt.

In dem untersuchten Material stellten wir ebenfalls die Erscheinung der Vergrösserung der Wirbelanzahl im Lendenabschnitt auf Kosten des Kreuzabschnittes fest. Dies betrifft einen Hirschbock (Kat. Nr. 2697), bei dem der erste Kreuzwirbel sich auf eine deutliche Weise an die Wirbel des Lendenabschnittes anähnelte. Dieser Wirbel behält eben seine absolute Selbständigkeit und mit dem eigentlichen Kreuzbein vereinigt er sich durch das Gelenk (Phot. 8 u. 9). Er verlor jedoch die Merkmale des ersten Kreuzwirbels nicht gänzlich, wenn auch nur in Hinsicht darauf, dass seine Querfortsätze als wenn eine Verlängerung der Kreuzbeinflügel sind. Es ist aber nicht genug damit, denn auf diesen Fortsätzen befinden sich Fragmente der Facies auriculares, die bekanntlich zur Vereinigung mit den Darmbeinen dienen. Diese Erscheinung bestimmen wir als Lumbalisatio des ersten Kreuzwirbels.

Es soll jedoch unterstrichen werden, dass diese Abwandlung einen deutlichen Kompensationscharakter hatte, denn es erfolgte bei dem beobachteten Hirschbock keine Vergrösserung der Wirbelanzahl des Lendenabschnittes bis sieben. Bei diesem Hirsch notierte man eine Verschiebung des ersten Lendenwirbels zum Brustabschnitt (Siehe weiter oben).

Im Kreuzschnitt, Pars sacralis, des Hirsches stellt man das Vorhandensein von 4 Wirbeln fest, die in das Kreuzbein verwachsen sind (Phot. 10). Bei einem jedoch enormen Teil der von uns beobachteten Individuen (27 Exemplare, darunter 21 Hirschböcke u. 6 Hirschkühe) wurde das Kreuzbein um den ersten Schwanzwirbel vergrössert, der sich mit 
diesem Bein vereinigte. Die Art der Vereinigung nimmt verschiedene Formen an.

In elf Fällen (bei 10 Hirschböcken im Alter von 2,5 bis über 12 Jahren und 1. achtjährigen Hirschkuh) stellten wir die Vereinigung des ersten Schwanzwirbels mit dem Kreuzbein vermittels aller Wirbelkomponenten fest, also ausser mit dem Wirbelkörper ebenfalls mit dem Dornfortsatz, den Gelenkfortsätzen und den Querfortsätzen, die zusammen eine unteilbare Gesamtheit bilden. Der von dem vorhergehenden Fortsatz etwas kleinere Dornfortsatz tritt in den Bestand der Crista sacralis media; die Gelenkfortsätze bilden die Cristae sacrales laterales, und die Querfortsätze die Margines laterales ossis sacri. Als einziges Anzeichen, das von der Verschiebung dieses Wirbels aus dem Schwanzabschnitt zeugen kann, können die beträchtlich vergrösserten Foramina sacralia zwischen dem letzten Kreuzwirbel und dem ersten Schwanzwirbel betrachtet werden (Phot. 11).

In fünf Fällen (4 Hirschböcke im Alter von 4 bis 10 Jahren und eine 9-jährige Hirschkuh) kam es noch nicht zu einer vollständigen Vereinigung des ersten Schwanzwirbels mit dem Kreuzbein, denn einige.Fragmente dieses Wirbels - meistenteils der Dornfortsatz, aber es kann ebenfalls ein einseitiger Gelenkfortsatz oder auch der Querfortsatz sein sind im Schwanzwirbel selbständig.

Bei elf Individuen ( 7 Hirschböcke von 2 bis über 12 Jahren und 4 Hirschkühe von 5 bis über 12 Jahren) unterscheidet sich der erste Schwanzwirbel deutlich vom letzten Kreuzwirbel trotz seiner Vereinigung vermittels des Wirbelkörpers, aber manchmal auch vermittels der Gelenkfortsätze oder auch der Querfortsätze jedoch beim Beibehalten einer deutlichen Grenze (Phot. 12).

Von der Erscheinung der Sacralisatio des ersten Schwanzwirbels kann man folglich nur in den Fällen der ersten beschriebenen Gruppe sprechen (10 Hirschböcke und 1 Hirschkuh), in der der verschobene Wirbel sich gänzlich an die Kreuzwirbel anglich und mit ihnen zusammen in den Bestand des unzertrennbaren Kreuzbeines trat. In den 16 übrigen Fällen, in denen der verschobene Wirbel eine teilweise Selbständigkeit bewahrte, haben wir mit einer Verbindung zu tun, die verschiedene Adaptationsformen des ersten Schwanzwirbels zum Kreuzabschnitt annimmt.

Diese Erscheinung ist bei Säugern recht gewöhnlich und kann ein Vorwand zu einer irrtümlichen Interpretation der Wirbelanzahl im Kreuzbein sein.

\section{SCHRIFTTUM}

1. A k a jevski A., 1962: Anatomia domašnih životnyh. Izd. Selhoz. Lit., 1-582. Moskva. 
2. Bronn H. G., 1874: Klassen und Ordnungen des Thier-Reichs, 6: 1-576. Leipzig u. Heidelberg.

3. Cuvier G., 1825: Recherches sur les ossements fossiles. Troisieme edition. Paris.

4. Kolda J., 1936: Srovnavaci anatomie zvirat domacich. Tiskla Novina, 1-914. Brno.

5. Pilarski W. \& R oskosz T., 1959: Beobachtungen über den Rumpfabschnitt der Wirbelsäule des Elches (Alces alces L.). Acta theriol., 3, 1: 1-16.

6. Poplewski R., 1948: Anatomia ssaków. Czytelnik, 2: 1-690. Stockholm.

7. Roskosz, T., 1962: Morphologie der Wirbelsäule des Wisents, Bison bonasus (L in n a e u s, 1758). Acta theriol., 6, 5. 113-162.

8. Roskosz T. \& Pytel S., 1966: Ein Versuch zur Bestimmung der Grösse des Wirbelkanals beim Rothirsch, Acta theriol., 11, 7: 269-280.

9. Wokken G., Glagolev P. \& Bogoljubski j S. 1961: Anatomia domašnih životnyh. Gos. Izd. „Vysšaja Škola”, 1: 1-391. Moskva.

Received January 22, 1966.

Institut für Anatomie der Tiere

an der Landwirtschaftlichen Hochschule,

Warszawa, ul. Grochowska 272.

Tadeusz ROSKOSZ i Stanisław PYTEL

ZMIENNOSĆ KRĘGOW I ŻEBER U JELENIA

Streszczenie

W oparciu o 42 kośćce jelenia (29 samców i 13 samic) autorzy dokonali obserwacji ilościowych i morfologicznych kręgów poszczególnych odcinków kręgosłupa. W szeregu przypadków odnotowano przesunięcia kręgów, połączone z upodobnieniem się ich do kręgów odcinków sąsiednich. Przesunięcia te są u jeleni nader częste i zachodzą niezależnie od wieku i płci zwierzęcia, jednakże udział samców jest tu zdecydowanie wiẹkszy (Tab. 1).

Pierwszą odnotowaną odmianą, która była udziałem 10 jeleni (okolo $23 \%$ badanego materiału) jest użebrowienie, costalisatio, pierwszego kręgu lędźwiowego. Polega ono na upodobnieniu się tego kręgu do kręgów piersiowych przez połączenie z żebrami bądź obustronnie ( 8 przypadków) - fot. $1-5$, bądź tylko jednej strony (2 przypadki) - fot. 6 - 7 .

Drugim rodzajem przesunięcia było upodobnienie się pierwszego kręgu krzyżowego do kręgów lędźwiowych, określone jako ulędźwowienie, lumbalisatio, pierwszego kręgu krzyżowego (Fot. 8-9).

Ostatnią wreszcie odnotowaną odmianą bylo ukrzyżowienie, sacralisatio, pierwszego kręgu ogonowego, polegające na całkowitym polączeniu się tego kręgu z kością krzyżową (Fot. 10-12). Była ona udzialem 11 osobników, co stanowi około $26 \%$ przebadanego materiału. 


\section{TAFELBESCHREIBUNG}

\section{Tafel XIII}

Costalisatio des ersten Lendenwirbels.

Phot. $1-\sigma$, Kat. Nr. 2697, Ansicht von oben.

Phot. 2 - \&, Kat. Nr. 383, Ansicht von oben.

Phot. $3-\sigma$, Kat. Nr. 362, Ansicht von vorn.

Phot. 4- $\sigma^{7}$, Kat. Nr. 381, Ansicht von oben.

Phot. $5-\sigma^{7}$, Kat. Nr. 375, Ansicht von hinten.

E.inseitige Costalisatio des ersten Lendenwirbels.

Phot. $6-\sigma^{7}$, Kat. Nr. 361, Ansicht von vorn.

Phot. $7-\sigma^{7}$, Kat. Nr. 364, Ansicht von hinten.

\section{Tafel XIV}

Lumbalisatio des ersten Kreuzwirbels.

Phot. $8-\sigma^{7}$, Kat. Nr. 2697, Ansicht von der Seite.

Phot. $9-\sigma^{7}$, Kat. Nr. 2697, Ansicht von unten.

Phot. 10 - Vierwirbeliges Kreuzbein, $\sigma^{7}$, Kat. Nr 363 - von der Seite.

Phot. 11 - Sacralisatio des ersten Schwanzwirbels, ơ, Kat. Nr. 364 Ansicht von der Seite.

Phot. 12 - Kreuzbein mit angeschlossenem ersten Schwanzwirbel, ðَ, Kat. Nr 2695, Ansicht von der Seite. 


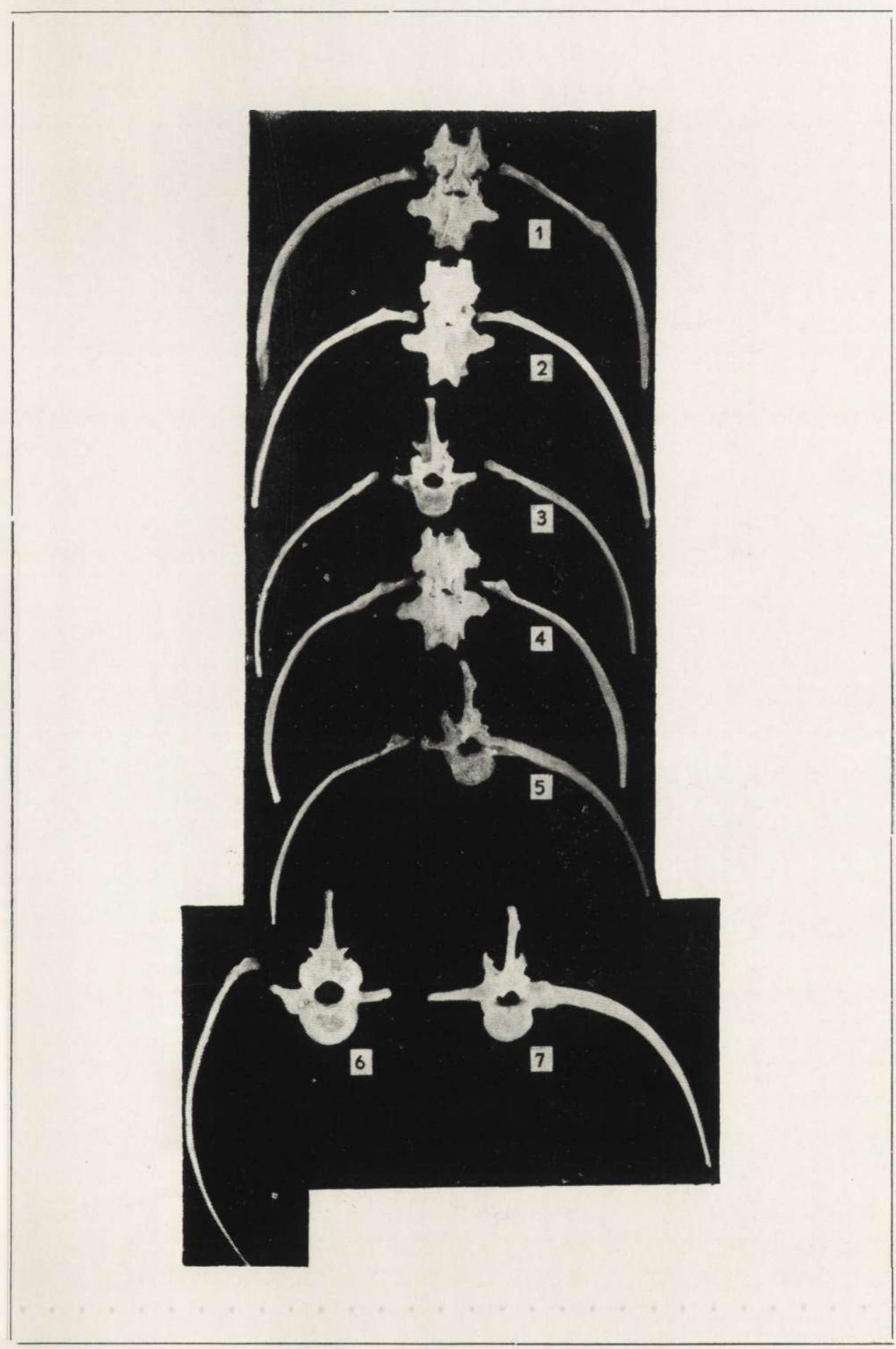

J. Roskosz \& S. Pytel 
ACTA THERIOLOGICA Vol. XI, 9.

BIBLIOTEKA

Instytutu Biologii Ssaków

Polskiej Akademii Nauk

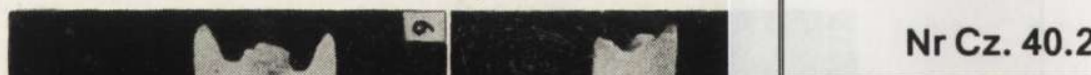

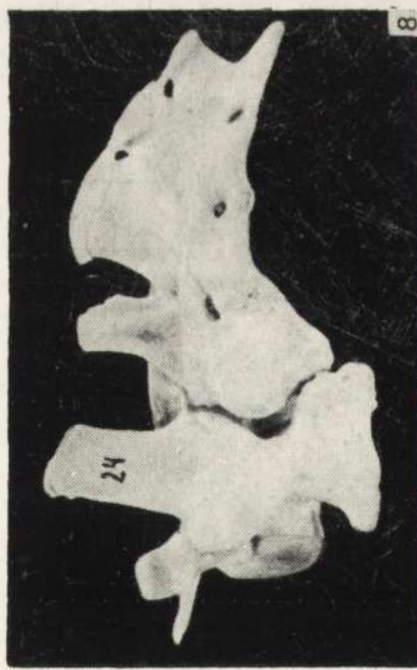

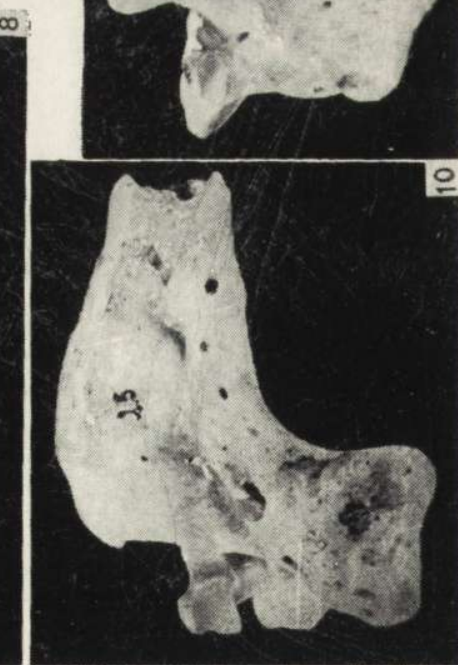

